\title{
Laser-induced assembly of gold nanoparticles into colloidal crystals
}

Nadine Harris, Mike J. Ford, Michael B. Cortie and Andrew M. McDonagh

Institute for Nanoscale Technology, University of Technology Sydney, PO Box 123,

Broadway, NSW 2007, Australia

E-mail: Mike.Ford@uts.edu.au 


\begin{abstract}
Three-dimensional, micron-sized colloidal crystal patterns have been synthesised directly from a gold nanoparticle/methyl methacrylate colloid using laser irradiation. A glass substrate was immersed within the colloid and a $500 \mathrm{~mW}$, $514 \mathrm{~nm}$ laser was used to irradiate the glass surface. Colloidal crystal patterns were created by translation of the glass substrate under the laser beam, after two minutes of irradiation, allowing a $4 \mathrm{~mm} \times 0.5 \mathrm{~mm}$ array of colloidal crystal patterns to be created. Through a series of control experiments and calculations we demonstrate that plasmon-induced, localized heating of the gold nanoparticles contributes to the mechanism behind this colloidal crystal formation phenomenon.
\end{abstract}

\title{
1. Introduction
}

For several years, considerable effort has been directed towards the synthesis of twoand three-dimensional colloidal crystals from gold nanoparticles. Potential applications of these structures include photonic crystals [1-3] waveguides [4] and active substrates for surface-enhanced Raman scattering (SERS) [5-11]. Templating [7, 12-15] and self-assembly [9, 16-20] techniques have been reported as routes to produce crystalline structures of gold nanoparticles. However, templating procedures require that a suitable template be produced prior to assembly, and the self-assembly technique requires that the nanoparticles and/or substrate be modified with functional groups to facilitate crystal assembly. Slow evaporation of solvent from a colloidal gold suspension [21-23] can also produce ordered structures although this more direct method provides little control over the deposition location. We report here a direct method for the controlled assembly of colloidal crystals from a gold sol that allows control of deposition location. We deposit micron-sized colloidal crystals onto glass surfaces using laser irradiation of a gold nanoparticle/methyl methacrylate (MMA) colloid without the use of templates or pre-functionalised nanoparticles or substrates. Deposition is controlled by the position of the laser beam, allowing patterns to be written onto glass by translation of the glass substrate.

\section{Methodology:}




\subsection{Synthesis of gold colloidal crystal nanostructures}

Dried oleylamine-stabilised gold nanoparticles with an average diameter of around $16 \mathrm{~nm}$ were prepared in toluene using the procedure described by Hiramatsu and Osterloh [21] and imaged with a Scanning Electron Microscope (SEM) (Supporting Information, Fig S1). Dynamic Light Scattering measurements of the gold nanoparticles show the average nanoparticle diameter to be $15.7 \mathrm{~nm}$ with a standard deviation of 6.3 (Supporting Information, Fig. S2). Methyl methacrylate (MMA) (Aldrich 99\%) was first washed with 10\% aqueous sodium hydroxide, then MilliQ water and dried with anhydrous sodium sulphate (Ajax) to remove the polymerisation inhibitor. Gold nanoparticle powder (4 mg) was then added to $10 \mathrm{ml}$ of MMA and sonicated for 1 minute to disperse the particles. The colloid was poured into a petri dish (5 $\mathrm{cm}$ diameter) containing a glass microscope slide so that the depth of the colloid was $\sim 5 \mathrm{~mm}$. The petri dish was mounted on an X-Y stage and a region of the glass slide was irradiated with a laser (514 nm continuous wave Coherent Innova 70 argon ion laser, $480 \mathrm{~mW}$ with a $1 \mathrm{~mm}$ spot size) for two minutes. The sample was then moved $0.5 \mathrm{~mm}$ and the procedure repeated until an array of dots covering around $4 \mathrm{~mm} \times 0.5 \mathrm{~mm}$ area was created. Figure 1 shows a schematic diagram of the experimental setup.

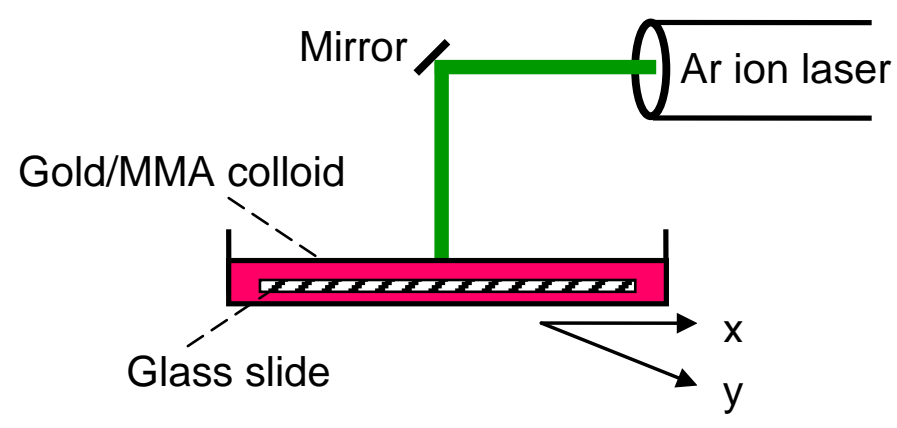

Figure 1. Schematic of the experimental setup. A $514 \mathrm{~nm}$ Argon ion laser irradiates a gold nanoparticle/MMA suspension. An $\mathrm{X}-\mathrm{Y}$ stage was used to translate the substrate.

\section{Results and Discussion:}




\subsection{Gold colloidal crystal nanostructures}

Figure 2 shows Environmental Scanning Electron Microscope (ESEM) and SEM images of an array of crescent-shaped dot structures formed on the glass slide after irradiation. Each crescent-shaped dot is formed from a single laser exposure of two minutes. Translation of the substrate using an X-Y stage allowed a $4 \mathrm{~mm} \times 0.5 \mathrm{~mm}$ array to be prepared (Figure 2a).

Energy Dispersive Spectroscopy (EDS) measurements on the crescent-shaped structures show silicon and gold signals assigned to the glass substrate and gold nanoparticles, respectively. (Supporting Information, Fig. S3). Figure 2a shows that each dot is approximately 300 microns in diameter, significantly smaller than the laser spot size $(1 \mathrm{~mm})$. The Gaussian beam profile of the laser presumably provides insufficient intensity towards the edges of the exposed region to cause formation of the colloidal crystals. The 300 micron structures are composed of smaller 2 - 3 micron-sized structures, Figures 2b-c. Higher magnification images reveal that these structures are composed of nanoparticles assembled in a close-packed structure, Figures 2d-f. Notably, these structures span several hundred particle diameters and exhibit very long range order with few defects. 

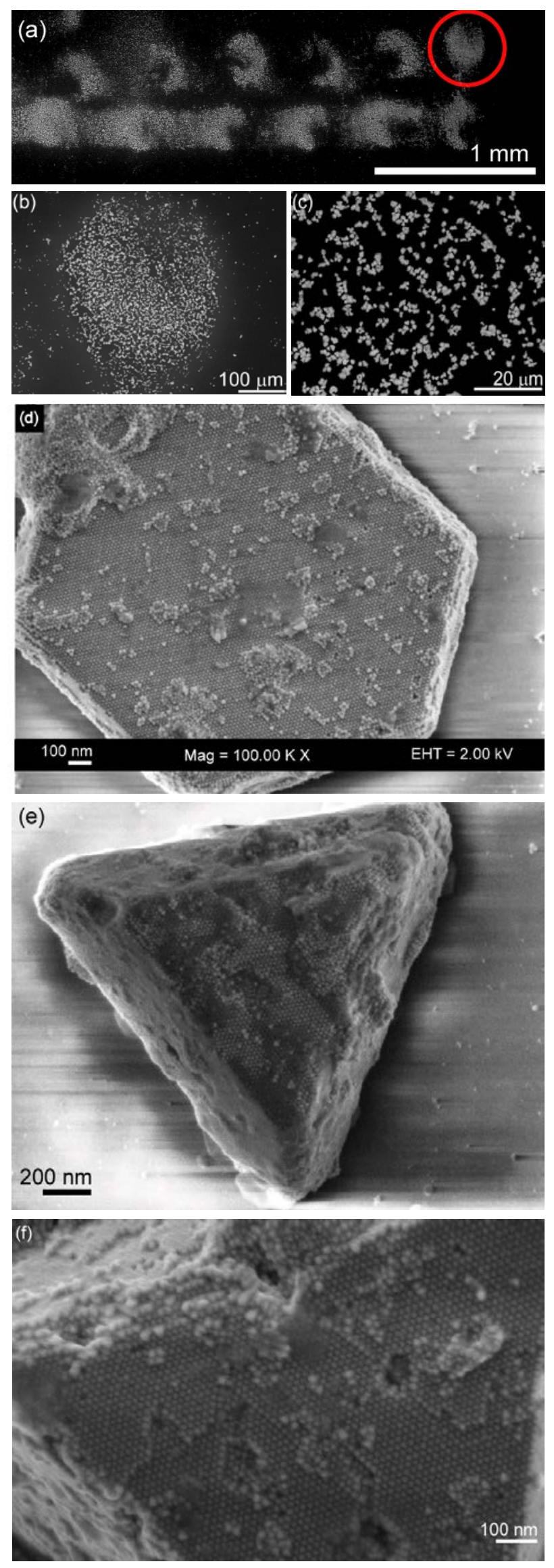
Figure 2. Images of laser-induced colloidal crystals deposited on a glass microscope slide. Figures 2a - 2c were imaged with a FEI XL30 ESEM operating in low-vacuum mode with an accelerating voltage of either $20 \mathrm{kV}$ or $30 \mathrm{kV}$ and a working distance of $10.4 \mathrm{~mm}$. Figures $2 \mathrm{~d}$ - 2f were imaged with a Zeiss Supra 55VP LEO SEM operating in high vacuum mode with an accelerating voltage of $2 \mathrm{kV}$, a working distance of $2 \mathrm{~mm}$ and an aperture size of $10 \mu \mathrm{m}$. The flaring, particularly in Figures $2 d$ and $2 e$ is due to charging of the glass slide while imaging. All samples were imaged on the glass microscope slide and remained uncoated. Figure 2b (magnified 387x), 2c (magnified 3098x), 2e (magnified $100,000 x$ ) and $2 f$ (magnified 200,000x).

\subsection{Control experiments}

The role of solvent was investigated by performing the laser irradiation experiments using gold nanoparticles resuspended in toluene (4 mg of dried gold particles in $10 \mathrm{ml}$ dried toluene). A representative image of the sample after laser irradiation is shown in Figure 3a. Large areas of nanoparticle aggregates of several layers are evident; in some areas, $500 \mathrm{~nm}$ thick aggregates were formed. Importantly, there is no evidence of close-ordered packing.

In experiments to prove thermal effects, glass substrates with droplets of the gold nanoparticle/MMA colloidal suspension were heated in an oven at $150^{\circ} \mathrm{C}$ for 20 minutes. Figure $3 \mathrm{~b}$ shows a SEM image of the resultant structures. The gold nanoparticles are attached to large blocks of material and no nanoparticle ordering is evident. Allowing droplets of gold nanoparticle/MMA colloid to evaporate at room temperature with no laser irradiation produces a different result, Figure 3c.

In this case large aggregates of gold nanoparticles form with a small number of areas exhibiting short-range ordering over an area of between 500 to $800 \mathrm{~nm}$. Moderate heating of the gold nanoparticle/MMA colloid in a test tube at $80^{\circ} \mathrm{C}$ in a water bath for 40 minutes gives a similar result, Figure 3d. 

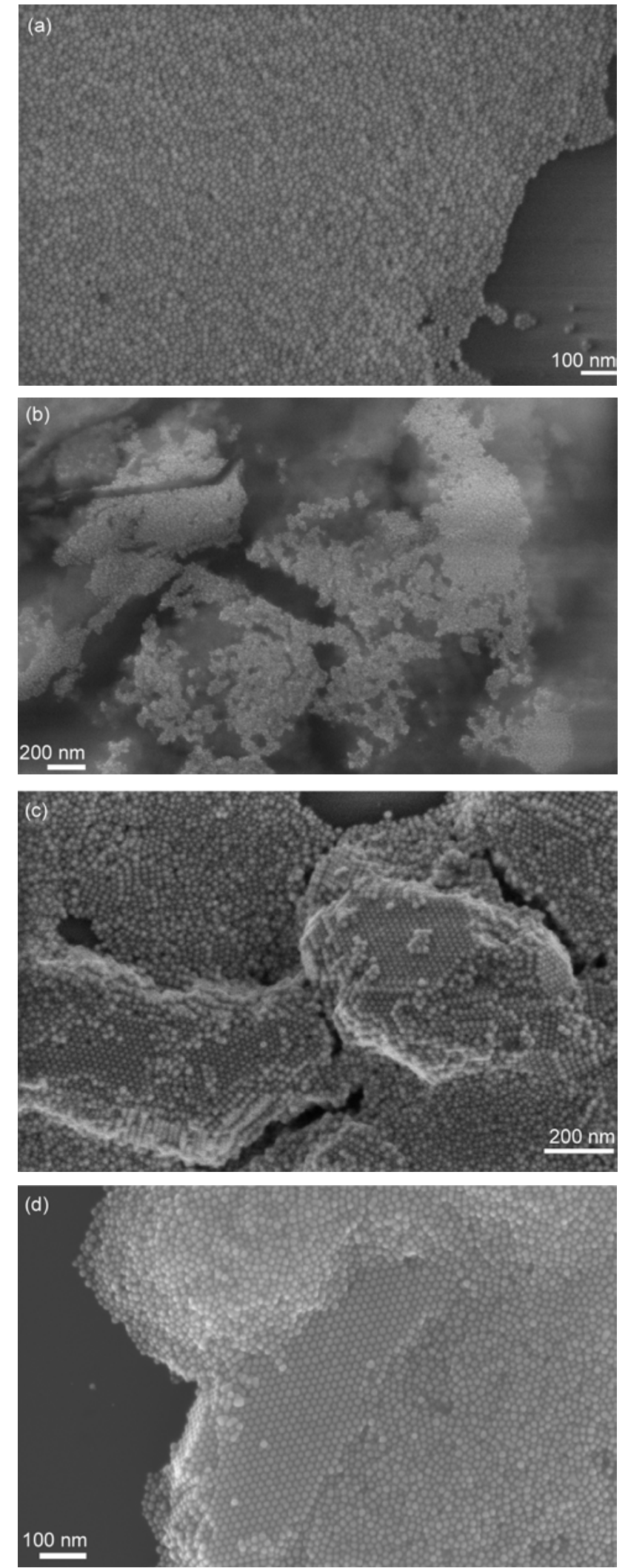

Figure 3. SEM images of (a) dried gold nanoparticles resuspended in toluene after laser irradiation and droplets of gold nanoparticle/MMA colloid (b) on a glass slide after 20 minutes in an oven at $150^{\circ} \mathrm{C}$, (c) evaporated on a microscope slide at room temperature and (d) heated to $80^{\circ} \mathrm{C}$ for 40 minutes. All figures 
(apart from Figure 3b) were imaged with a Zeiss Supra 55VP LEO SEM operating in high vacuum mode with an accelrating voltage of $2 \mathrm{kV}$, a working distance of $2 \mathrm{~mm}$ and an aperture size of $10 \mu \mathrm{m}$. For Figure 3b the SEM was operating in low-vacuum mode with an accelerating voltage of $20 \mathrm{kV}$, a working distance of $5 \mathrm{~mm}$ and an aperture size of $30 \mu \mathrm{m}$. Specimens were uncoated.

Although colloidal crystals formed at room temperature there was significantly less long-range ordering. Heating the colloidal/MMA suspension in an oven did not produce ordered structures. One explanation for this observation is that the MMA rapidly polymerizes (the large blocks observed in Figure $3 \mathrm{~b}$ ) and fixes the position of the nanoparticles prohibiting ordering into crystalline structures. The fact that only limited ordering occurs for the evaporated and moderately heated sample would appear to rule out a simple temperature driven annealing processes as the ordering mechanism. The laser irradiated samples display ordering on a scale that is not achieved in these experiments suggests that localised plasmonic heating plays a crucial role. These experiments also show that MMA is critical to the formation of colloidal crystals. Chemical modification of the MMA induced by localised laser heating is a possible mechanism driving the ordering.

\subsection{Discussion}

No gold particles were observed in areas that were not exposed to laser irradiation. We propose, therefore, that ordering of the nanoparticles is facilitated by laserinduced heating. Localised heating of gold nanoparticles is a well-established effect [24-27]. Gold nanoparticles in the size range of $20 \mathrm{~nm}$ to about $100 \mathrm{~nm}$ show a pronounced plasmon resonance at approximately $520 \mathrm{~nm}$, where they absorb incident light up to several times their geometric cross-section. The absorption efficiency, $Q_{a b s}$, of a $16 \mathrm{~nm}$ diameter spherical gold particle in MMA was calculated using Mie theory [28] (Supporting information, Fig. S4). A frequency independent refractive index of 1.414 [29] was assumed for MMA. The $Q_{a b s}$ peak was calculated to be at $532 \mathrm{~nm}$ where the particle absorbs 1.43 times its geometric cross-section. At our laser wavelength of $514 \mathrm{~nm} Q_{a b s}$ is 0.96. The absorbance spectrum of the gold nanoparticle/MMA colloid can also be measured experimentally. The measured 
absorbance peak is 0.46 at approximately $602 \mathrm{~nm}$. This corresponds to a nanoparticle $Q_{a b s}$ of 0.47 . The difference between the measured $Q_{a b s}(0.47)$ and the calculated $Q_{a b s}$ (1.43) results from particle aggregation when the dried gold nanoparticles are resuspended in MMA. This causes a red shift and broadening of the absorbance spectrum (Supporting Information, Fig. S5). The original gold/toluene colloid produces a very narrow absorbance peak with a resonance of $525 \mathrm{~nm}$ (Supporting Information, Fig. S6).

The following calculation shows that a significant degree of heating of gold nanoparticles can be achieved through laser irradiation. We have previously reported a method to calculate the increase in temperature of a nanoparticle induced by the absorption of light [24]. The absorbed radiation is converted to thermal energy thereby heating the nanoparticle and surrounding liquid. The heat transfer rate, $P$, measured in watts, can be calculated from,

$$
P=I C_{a b s}
$$

where $I$ is the laser intensity and $C_{a b s}$ is the particle absorption cross-section, equal to absorption efficiency, $Q_{a b s}$ multiplied by the particle cross-sectional area. $P$ is calculated to be $1.18^{-10} \mathrm{~W}$ for the present laser intensity of approximately $611 \mathrm{~kW} / \mathrm{m}^{2}$, $Q_{a b s}$ of 0.96 at $514 \mathrm{~nm}$ and $16 \mathrm{~nm}$ diameter particles. The temperature of an individual nanoparticle during laser irradiation can be calculated using a convective heat transfer model [24],

$$
P=h 4 \pi r^{2} \delta T
$$

where $h$ is the heat transfer coefficient, $r$ the nanoparticle radius and $\delta T$ the change in nanoparticle temperature. Using a value of $h$ of $240 \mathrm{~W} \mathrm{~m}^{-2} \mathrm{~K}^{-1}$ corresponding to the heating of a nanofluid for 500 seconds [30] $\delta T$ is calculated to be $\approx 610 \mathrm{~K}$, a significant increase in particle temperature.

\subsection{UV-Vis absorption spectrum}

The UV-Vis absorption spectrum of the colloidal crystal is shown in Figure 4. It can be seen that the observed spectrum is relatively flat and there is no plasmon resonance band in the vicinity of $602 \mathrm{~nm}$ indicative of isolated gold nanoparticles in MMA (Supporting Information, Fig. S5). Although the SEM images in Figure 2 show welldefined gold nanoparticles constituting the colloidal crystal, this flat absorbance 
spectrum shows that the gold particles are effectively in contact, or close enough to each other that their optical responses are heavily coupled leading to a loss of the single-particle plasmon effect.

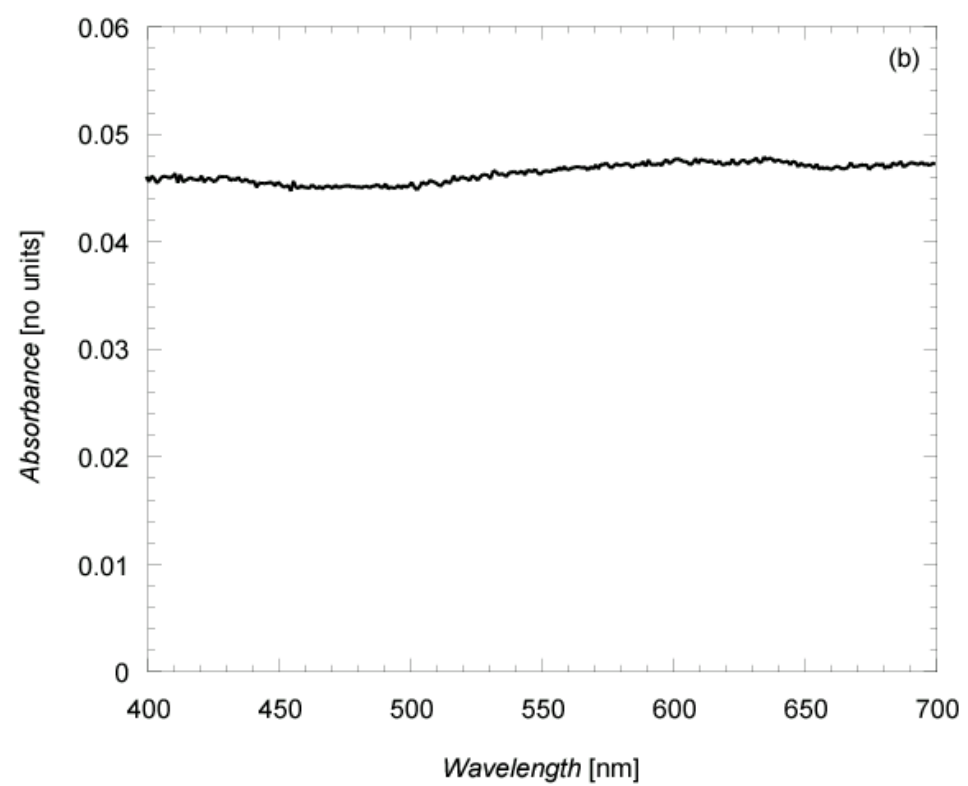

Figure 4. UV-Vis absorbance spectrum of the $4 \mathrm{~mm} \times 0.5 \mathrm{~mm}$ array of colloidal crystal structures on the glass slide. Obtained with a Cary $5 \mathrm{E}$ UV-Vis-NIR Spectrophotometer using a plain microscope slide for baseline correction

\section{Conclusion:}

In summary, we have developed a facile method to synthesise three-dimensional, micron-sized gold colloidal crystals by laser-induced heating of gold nanoparticles suspended in MMA. This technique allows the user to write colloidal crystal patterns by moving a laser beam over a glass substrate. While the mechanism behind this phenomenon is still under investigation, it is believed that the laser-induced plasmonic heating of the gold nanoparticles is an inherent requirement within the formation process.

\section{Acknowledgments:}

This work was supported by the Australian Research Council (ARC) and the University of Technology (UTS), Sydney. The authors thank Katie McBean and 
Richard Wuhrer of the Microstructural Analysis Unit at UTS for their assistance with scanning electron microscope imaging as well as Åsa Jamting from the National Measurement Institute, Lindfield, Sydney for her assistance with particle sizing measurements. 


\section{References:}

1. Yablonovitch E 1987 Phys. Rev. Lett. 582059.

2. $\quad$ Takeda S and Wiltzius P 2006 Chem. Mater. 185643.

3. $\quad$ Tarhan I I and Watson G H 1996 Phys. Rev. Lett. 76315.

4. Zhang X, Sun B, and Friend R H 2006 Nano Lett. 6651.

5. $\quad$ Griffith F R, et al. 1995 Science. 2671629.

6. $\quad$ Nie S and Emroy S R 1997 Science. 2751102.

7. Tessier P M, Velec O D, Kalambur A T, Rabolt J F, Lenhoff A M, and Kaler E M 2000 J. Am. Chem. Soc. 1229554.

8. Kubo S, Gu Z-Z, Tryk D A, Ohko Y, Sato O, and Fujishima A 2002 Langmuir. 185043.

9. Lu L, Randjelovic I, Capek R, Gaponik N, Yang J, Zhang H, and Eychmuller A 2005 Chem. Mater. 175731.

10. Brolo A G, Arctander E, Gordon R, Leathem B, and Kavanagh K L 2004 Nano Lett. 42015.

11. Dick L A, McFarland A D, Haynes C L, and Duyne R P V 2002 J. Phys. Chem. B. 106853.

12. Dong W, Dong H, Wang Z, Zhan P, Yu Z, Zhao X, Zhu Y, and Ming N 2006 Adv. Mater. 18755.

13. Juillerat F, Solak H H, Bowen P, and Hofmann H 2005 Nanotechnology. 16 1311.

14. Yi K C, Mendieta V S, Castanares R L, Meldrum F C, Wu C, and Fendler J H 1995 J. Phys. Chem. 999869.

15. Liang Z, Susha A S, and Caruso F 2002 Adv. Mater. 141097.

16. Wang S, Sato S, and Kimura K 2003 Chem. Mater. 152445.

17. Huang H-Y, Chen W-F, and Kuo P-L 2005 J. Phys. Chem. B. 10924288.

18. $\quad$ Lin X M, Wang G M, Sorensen C M, and Klabunde K J 1999 103. 26.

19. Zhao S-Y, Wang S, and Kimura K 2004 Langmuir. 201977.

20. Osifchin R G, Mahoney W J, Bielefeld J D, and Andres R P 1995 Superlattices and Microstructures. 18283.

21. Hiramatsu H and Osterloh F E 2004 Chem. Mater. 162509.

22. Brust M, Brethell D, Schiffrin D J, and Kiely C J 1995 Adv. Mater. 7795. 
23. Stoeva S, Klabunde K J, Sorensen C M, and Dragieva I 2002 J. Am. Chem. Soc. 1242305.

24. Harris N, Ford M J, and Cortie M B 2006 J. Phys. Chem. B. 11010701.

25. Pissuwan D, Valenzuela S M, and Cortie M B 2006 Trends in Biotechnology. 2462.

26. Hu M and Hartland G V 2002 J. Phys. Chem. B. 1067029.

27. Govorov A O, Zhang W, Skeini T, Richardson H, Lee J, and Kotov N 2006 Nanoscale Res. Lett. 184.

28. Mie G 1908 Ann. Phys. 25377.

29. Sunkara H B, Jethmalani J M, and Ford W T 1994 Chem. Mater. 6362.

30. Wen D and Ding Y 2006 IEEE Transactions on Nanotechnology. 5220. 